Musées, Patrimoine et Culture scientifiques et techniques

$146 \mid 2013$

mars-avril 2013

\title{
La constitution et la valorisation d'archives orales dans un musée scientifique et technique
}

Marianne Lombardi et Emmanuelle Cournarie

\section{OpenEdition \\ Journals}

Édition électronique

URL : http://journals.openedition.org/ocim/1199

DOI : 10.4000/ocim. 1199

ISSN : 2108-646X

Éditeur

OCIM

Édition imprimée

Date de publication : 1 mars 2013

Pagination : 12-18

ISSN : 0994-1908

Référence électronique

Marianne Lombardi et Emmanuelle Cournarie, «La constitution et la valorisation d'archives orales dans un musée scientifique et technique », La Lettre de I'OCIM [En ligne], 146 | 2013, mis en ligne le 01 mars 2015, consulté le 01 mai 2019. URL : http://journals.openedition.org/ocim/1199 ; DOI : 10.4000/ ocim. 1199 


\section{La constitution et la valorisation $d$ 'archives orales dans un musée scientifique et technique}

\section{Marianne Lombardi et Emmanuelle Cournarie *}

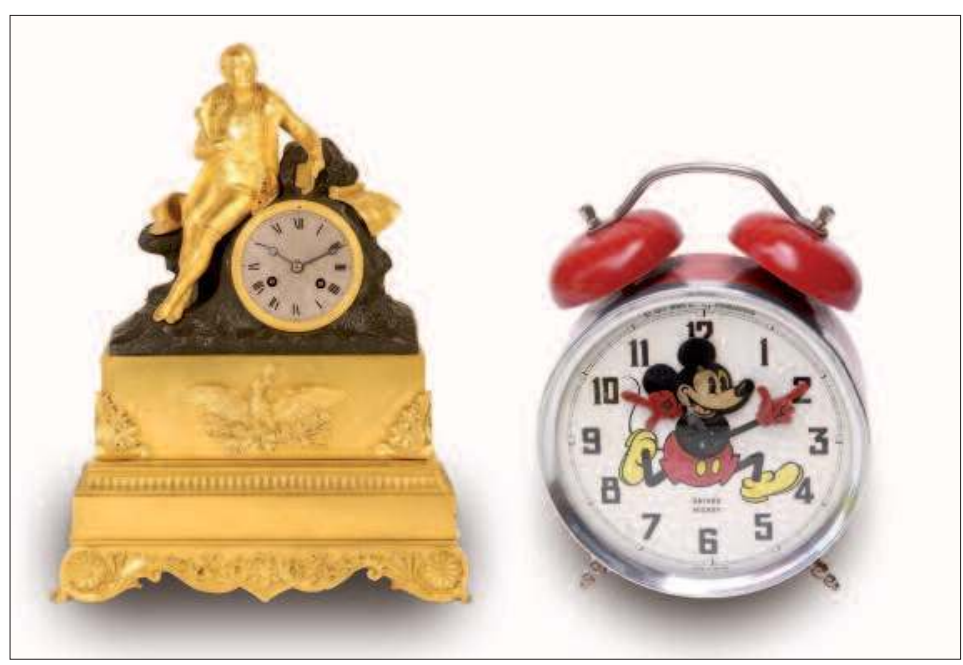

La pendule de cheminée Honoré Pons (XIXe siècle) et le réveil animé Bayard (1989) : le savoir-faire des horlogers aliermontais (c) Marianne Lombardi

* Marianne Lombardi est assistante qualifiée de conservation, directrice du musée de l'Horlogerie

à Saint-Nicolas-d'Aliermont Emmanuelle Cournarie est docteur d'État en socioanthropologie, chercheur au laboratoire de Sociologie et d'Anthropologie de l'université de Franche-Comté
Recueillir les éléments du contexte technique, historique et social de la production horlogère à Saint-Nicolas-d'Aliermont auprès des anciens salariés et leurs familles pour en assurer la sauvegarde puis intégrer les résultats de cette recherche au discours du musée : tels étaient les principaux objectifs du travail de collecte de mémoire orale présenté ici par les responsables du projet.

Le musée de l'Horlogerie de Saint-Nicolas-d'Aliermont (4 000 habitants, Haute-Normandie) retrace l'histoire de l'horlogerie et de la précision, savoir-faire original de la ville, du début du XVIIIe siècle à nos jours.

Initiée en 1725, l'aventure horlogère aliermontaise s'est poursuivie pendant deux siècles et se prolonge aujourd'hui à travers la microtechnique et la mécanique de précision. Production régionale, «l'élégante » de Saint-Nicolas est à l'origine de l'horlogerie domestique régionale. Les pendules de cheminée signées Honoré Pons puis les chronomètres de marine entretiennent ce savoir-faire basé sur la qualité et le travail de précision. Le réveil, produit en série par la société Bayard (1867-1989), fonde ensuite la renommée internationale de la commune. Dans les années 1970, de profondes mutations industrielles interviennent : les usines horlogères commencent, soit à fermer leurs portes, soit à diversifier leurs productions, voire à se reconvertir totalement. De nos jours Saint-Nicolas-d'Aliermont conserve une activité industrielle de mécanique de précision, directe héritière des manufactures horlogères de jadis. Cette activité industrielle a marqué le paysage de la commune qui possède un riche patrimoine industriel, vecteur 
de mémoire sociale : cités ouvrières (dont une réalisée par Le Corbusier), usines, maisons traditionnelles d'horlogers...

Le musée a été fondé en 1981 par des dirigeants et employés des usines horlogères et mécaniques de la ville, réunis dans l'Association d'Horlogerie Aliermontaise. L'association a constitué une collection exceptionnelle d'objets horlogers et mécaniques du XVIIe siècle à nos jours complétée par des fonds d'archives, de photographies et une bibliothèque spécialisée. En 2006, elle a donné ses collections à la ville qui a construit un musée moderne, reconnu « Musée de France ». Il est administré en régie directe par la municipalité fortement impliquée dans son développement.

\section{La constitution d'archives orales}

\section{Les enjeux}

Le personnel travaille en pleine collaboration avec les membres de l'association qui ont encore aujourd'hui toute leur place dans le musée : ils font fonctionner un certain nombre de pièces exposées, aident à la réalisation des fiches d'inventaire et possèdent un atelier opérationnel au cœur du bâtiment.

Très vite, la problématique de la conservation de ce savoir-faire se pose : savoir technique, certes, mais aussi témoignages qui permettent d'éclairer le contexte de production des différents objets exposés. L'atelier actuel, qui accueille quelques membres plus jeunes, répond encore à la transmission du savoir-faire technique en horlogerie, mais tout le contexte historique et social de la production horlogère et mécanique du $\mathrm{XX}^{\mathrm{e}}$ siècle est en cours de disparition. Pour ce dernier, seul un travail exhaustif et systématique de collecte de la mémoire peut permettre une véritable sauvegarde et une transmission effective.

Lors de la redéfinition du parcours du musée au sein du nouveau bâtiment, entre 2005 et 2007, la place de cette mémoire a été pensée. Mélanie Thomas, alors directrice du musée, met en place la première phase d'un projet de trois ans : la constitution d'un fonds d'archives orales en vue de sa conservation, de sa valorisation et de sa restitution par le biais d'une intégration au discours du musée.

Afin de récolter des informations exploitables, la collectivité a lancé un appel d'offres national au printemps 2009. Le cahier des charges demandait notamment : - la mise en œeuvre de protocoles propres aux disciplines anthropologiques et ethnologiques ;

- une analyse profonde de la thématique de l'horlogerie et de la micromécanique ;
- la collecte de témoignages, enregistrés et filmés pour certains ;

- la transcription complète des entretiens et le rendu d'une synthèse ;

- la communication des travaux en cours lors de rencontres avec la population locale ;

- le travail en partenariat avec un photographe (missionné par la municipalité).

Sur le plan scientifique, l'étude avait notamment pour objectif d'approfondir les fonctionnements internes et externes des usines horlogères et mécaniques. Elle devait appréhender les aspects techniques des métiers exercés au sein des usines, l'organisation du travail, mais tenir aussi compte des aspects sociologiques.

\section{Quelques exemples de sujets abordés dans la collecte d'archives orales}

Fonctionnements internes :

- les conditions de travail

- l'organisation/la répartition du travail et son évolution au cours de la carrière

- les pratiques syndicales,

- la notion de paternalisme, les relations hiérarchiques,

- les moments de crise, la question de la reconversion

- les différents métiers qui existaient au sein de l'usine

- les femmes à l'usine : quelle place ? quel(s) métier(s)?

- les notions d'émulation, de solidarité, de réseaux

- l'esprit d'entreprise

- l'identité de l'horloger aliermontais

- la perception et la relation au temps sont-elles particulières ?

- la chaîne opératoire, les savoir-faire et leurs évolutions, l'outillage, le vocabulaire

\section{Fonctionnements externes :}

- le lien entre la vie de l'usine et les événements, fêtes et cérémonies

- la scolarisation et l'entrée à l'usine

- les loisirs hors du temps de travail

- la place de l'usine dans la vie familiale

La méthodologie utilisée

Suite à l'appel d'offres, la collectivité a choisi la proposition d'Emmanuelle Cournarie, à l'époque doctorante 
au laboratoire de Sociologie et d'Anthropologie de l'université de Franche-Comté (LASA-UFC), spécialisée dans le champ de la sociologie des organisations, des entreprises et du travail. Sa thèse porte sur l'industrie horlogère bisontine, elle a donc une bonne connaissance du milieu et du vocabulaire horloger et mécanique et une vision étendue profitable à des comparaisons entre notre ville et la grande région horlogère française, la Franche-Comté.

La mission sociologique a duré six mois, dont trois mois sur le terrain durant l'automne 2009. Une phase exploratoire a été organisée en amont pour prendre contact avec le terrain, le public et élaborer la problématique de recherche. Lorsque l'on cherche à recueillir des données empiriques, a fortiori par le biais de témoignages, les dangers sont nombreux. L'accès au terrain est difficile et pose de nombreuses questions : quels thèmes aborder et dans quel(s) but(s) ? Qui interroger? Que faire des données recueillies? Comment en extraire des éléments mobilisables dans une dynamique de valorisation du patrimoine ? Autant de problèmes que la démarche de recherche en sciences sociales vise à résoudre en suivant une méthode stricte.

Le socio-anthropologue n'investit pas le terrain sans avoir suivi plusieurs étapes le conduisant à recueillir les données in situ. Partant d'une question de départ relativement large, il entre dans une phase d'exploration faite de lectures et d'entretiens exploratoires. Dans le cadre de cette collecte, l'exploration s'est construite autour du questionnement du personnel muséal sur les savoirs et savoir-faire techniques propres au territoire, les modalités de production des objets exposés dans le musée de l'horlogerie, le contexte

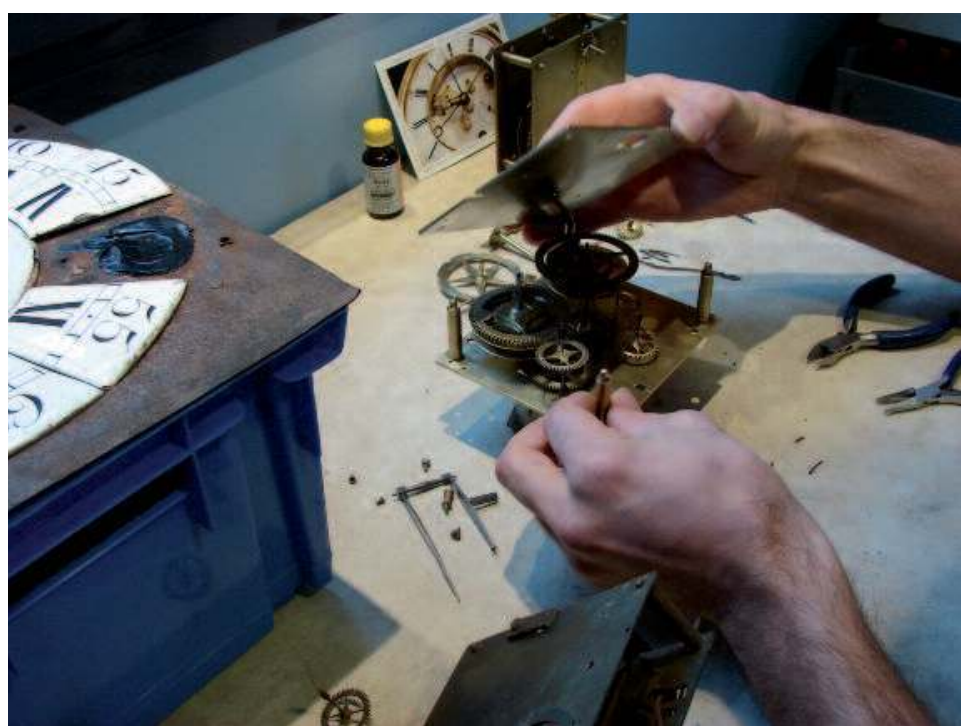

L'atelier d'horlogerie du musée de Saint-Nicolas-d'Aliermont () Marianne Lombardi historique et social de la production, les modalités de reconversion de l'industrie locale au tournant des années 1980. Une revue de littérature portant sur les thèmes précités a été effectuée à partir d'une documentation riche fournie par les agents du musée. Ces lectures ont été complétées par quatre entretiens exploratoires réalisés avec des personnes « ressources », c'est-à-dire ayant une bonne connaissance du territoire industriel et ses spécificités.

Suite à cette phase exploratoire, le socio-anthropologue a construit une problématique et proposé des hypothèses qu'il a cherché à confirmer ou infirmer grâce aux matériaux recueillis sur le terrain. Ici, il s'agissait de comprendre comment le système industriel étudié est parvenu à se pérenniser durant plusieurs siècles puis comment il s'est transformé en un système centré sur la micromécanique. Pour répondre à cette interrogation, une hypothèse a été proposée stipulant que la stabilité de l'industrie aliermontaise d'avant crise reposait à la fois sur une organisation spécifique de la production et sur une culture professionnelle fortement cohésive. En travaillant à partir des concepts d'« organisation » et de « culture » les objectifs de la recherche pure et de la recherche appliquée ont été combinés. Car pour approfondir les connaissances fondamentales en matière d'« organisation productive traditionnelle » et de " culture professionnelle », ont été identifiés, décrits et analysés les savoirs et savoir-faire techniques propres au territoire, les modalités de production des objets exposés dans le musée de l'horlogerie, le contexte historique et social de la production. Ces éléments immatériels, servant la recherche fondamentale, étaient également ceux que souhaitait recueillir l'équipe muséale dans le cadre de sa collecte de mémoire orale.

\section{Une base de données papier et multimédia}

Après avoir réalisé le canevas d'entretiens, E. Cournarie a rencontré chaque volontaire, de préférence à domicile, accompagnée d'un photographe missionné par la ville. Le « corpus » des personnes interrogées s'est construit au fur et à mesure des rencontres, chaque personne nous conseillant de parler à telle autre pour approfondir des aspects précis. Au final, 36 entretiens ont été menés, parfois deux personnes (conjoints, sœurs, parents) ont participé au même entretien. Nous ne souhaitions pas mener des entretiens doubles mais certaines personnes ne souhaitaient pas parler seules (peur d'oublier des choses, difficulté à être le centre d'attention), et dans d'autres cas le conjoint s'impose plus ou moins, la rencontre ayant lieu à domicile. Parfois, le fait d'être à deux enrichit vraiment le propos (complément, relance), mais dans au 
Bien qu'elles soient mobilisées pour effectuer des tâches subalternes de production ou confinées dans le domaine administratif, les femmes occupent une place prépondérante dans le système productif aliermontais et représentent en nombre le groupe le plus important au sein de la population ouvrière locale. Les entreprises de production, grandes et moyennes, mobilisent une charge de main-d'œuvre non qualifiée conséquente, sur laquelle repose en grande partie la productivité de l'industrie locale. Et si les femmes n'ont guère de pouvoir ni d'avantages, elles trouvent des compensations par le biais du collectif de travail et dans le statut même de salariées.

Dans notre échantillon, 9 femmes, dont 5 ouvrières spécialisées et 4 employées administratives ont été interrogées. Les employées administratives sont issues de familles rurales, disposant de moyens limités mais avec des fratries relativement petites. Ces secrétaires, comptables ou sténodactylos ont en effet entre 1 et 3 frères et sœurs. Les ouvrières spécialisées sont issues de familles rurales également, plutôt modestes, mais avec des fratries importantes, comptant jusquà 13 enfants. Lorsque les fratries sont moins importantes, il manque généralement un des deux parents, ce qui conduit les jeunes filles à travailler très tôt. Les témoignages démontrent que l'entrée au travail se fait aux alentours de 14 ans, dans le but de contribuer à l'économie de la maisonnée. « Nous sommes une famille de 13 enfants, et je suis la $5^{e}$. Quand on est devenus tous grands, à part mes frères aînés qui ont pu faire un tout petit peu d'études, nous on a été obligés d'aller travailler, ça faisait partie de la famille, fallait faire rentrer de l'argent, c'était tout à fait normal. Alors moi j'avais 15 ans quand je suis entrée chez Bayard " (témoignage d'une ancienne ouvrière de l'entreprise Bayard).

Les ouvrières qui travaillaient dans les entreprises locales occupaient des postes divers, soit dans des ateliers de mécanique, soit sur des chaînes d'assemblage. Lorsqu'elles travaillaient à la mécanique, les femmes se plaignent plutôt des conditions de travail difficiles en termes de saleté, de bruit, et de sécurité, alors que celles qui étaient à la chaîne insistent sur les cadences de travail. «Moi jétais à l'atelier, ils m'avaient mis sur un tour. Il y a un jeune qui était parti au régiment, il travaillait sur les tours à huile, et puis on miavait mis là en remplacement. Mais alors c'était sale, il fallait mettre des chiffons, tout ça, vous en aviez plein la figure, plein les vêtements. C'était une fraiseuse qu'on appelait ça » (témoignage d'une ancienne ouvrière de l'entreprise Bayard).
Malgré les conditions de travail difficiles, les longues journées de travail (de 7 h 00 à 12 h 00 et de 13 h 30 à $18 \mathrm{~h} 00$ ), les risques et les accidents du travail (écrasement des doigts, coupures...), les femmes sont très nostalgiques du temps où elles travaillaient à l'usine, et ne se focalisent pas sur les difficultés liées au travail, mais plutôt sur ce que le travail leur apporte. «Chez Lemaignien ça faisait 20 ans que jyy étais et on se connaissait bien. C'était tout dans le même montage alors forcément... Bon on faisait des blagues à cette époque-là, mais sans arrière-pensées. Tout le monde était... Oh on s'entendait bien! » (témoignage d'une ancienne ouvrière de l'entreprise LLM).

Chez les ouvrières, le travail - que ce soit aux chaines où le rythme est imposé par la direction ou lorsqu'elles exécutent des tâches, notamment dans les ateliers de mécanique, où elles sont chronométrées - est évoqué d'abord en termes d'« efficacité » ou de "rapidité » dans l'exécution des gestes. Les postes qu'elles occupent sont soumis à des rythmes, des temps qu'elles doivent respecter, souvent dans le but de bénéficier de primes de rendement.

Les travaux sociologiques réalisés sur les ouvrières démontrent que ces dernières sont systématiquement confinées à des travaux pénibles à cycles courts,c'est-à-dire avec une répétition de gestes similaires sur des temps très réduits. Si les qualifications des ouvrières ne sont pas reconnues, malgré leur dextérité, leur vitesse, c'est que leurs aptitudes sont considérées comme provenant de la sphère domestique, acquises sans apprentissage formalisé. Les qualifications habiles, parfois hautement complexes, requises pour ces travaux, sont tenues pour des dons de nature ou des qualifications sexuelles générales, payées comme telles aux prix les plus bas.

Ce qui semble prépondérant chez les ouvrières, ce sont les arts d'ambiance, les valeurs de collectif et de camaraderie, les blagues au travail. Il faut souligner tout de même que la plupart des femmes que nous avons interrogées travaillaient lorsqu'elles étaient jeunes, généralement avant de se marier. Peut-être y a-til ici un effet générationnel ? Mais pour avoir interrogé des ouvrières de l'industrie bisontine, il nous semble que la nostalgie est beaucoup plus présente à SaintNicolas-d'Aliermont qu'ailleurs. 
moins deux rencontres, cela nuit vraiment à la clarté de l'entretien (personnes qui se coupent la parole constamment).

Au préalable, E. Cournarie a demandé à chaque personne interrogée de choisir un objet évocateur, point de départ de l'entretien et objet mis en avant sur le portrait photographique.

Chaque entretien a été enregistré, transcrit puis codé selon les thématiques envisagées dans l'appel d'offres. Le but était de fournir au musée des documents numériques facilement exploitables par la suite.

Nous sommes également retournés chez trois personnes, sélectionnées pour leur représentativité, pour réaliser un deuxième entretien thématisé et filmé afin de produire un document vidéo de quelques minutes. Ces petits films ont surtout été réutilisés lors des soirées de compte-rendu à la population ainsi que lors d'expositions temporaires.

Tout au long du processus, une grande attention a été apportée sur l'implication des personnes interrogées et le respect de leurs volontés (anonymat pour certains, non divulgation de certains passages des entretiens). Des contrats de cessions des droits d'auteurs et des droits à l'image ont été signés par chaque participant afin de rendre exploitables toutes les données recueillies pour de futures publications, expositions... Cet aspect des choses est toujours un peu difficile pour les volontaires, qui craignent de mauvaises interprétations de leurs propos et hésitent à signer un document à l'aspect et au contenu, très juridique. Il a donc fallu user d'une grande pédagogie (assurance de leur faire relire les textes dans lesquels leurs noms apparaissent...), et parfois solliciter l'intervention de «personnes ressources " (élus, personnalités locales). En définitive, seules 4 personnes ont refusé d'être photographiées, tout en acceptant quand même de témoigner. Une attention particulière a été apportée à la restitution à la population, ce qui a aussi permis de limiter les inquiétudes. Chaque personne a ainsi reçu une copie de l'entretien, un tirage de sa photographie, des invitations pour assister au bilan final ainsi qu'à l'exposition des portraits photographiques accompagnés de citations présentée en mars 2010 au musée.

Un rapport final de 80 pages, a été rédigé et remis au musée, il contient un résumé du projet d'étude, une présentation de la méthodologie de l'enquête, un bref rappel de l'histoire horlogère aliermontaise et l'analyse de E. Cournarie présentant les caractéristiques de l'industrie locale d'avant crise, les changements au tournant des années 1980 et les caractéristiques de la population horlogère de l'Aliermont. La vocation de ce document n'était pas d'être accessible au grand public, mais bien de servir de base pour les projets futurs du musée.

\section{Des relais scientifiques et financiers indispensables}

Le patrimoine immatériel fait l'objet depuis quelques années de nombreuses études et a acquis une reconnaissance internationale au sein de l'Unesco. Afin de s'inscrire dans une démarche scientifique éprouvée, le personnel du musée a demandé conseil à des relais régionaux pour la mise en place du projet et pour organiser le recrutement d'Emmanuelle Cournarie.

Les principaux relais ont été les secteurs musées et ethnologie de la DRAC de Haute-Normandie, ainsi que le service Monuments historiques - Musées - Patrimoine immatériel de la Région Haute-Normandie, très impliqué dans la promotion de la mémoire orale.

Ces structures ont financé fortement les différentes phases du projet, ce qui a soutenu l'effort de la municipalité de Saint-Nicolas-d'Aliermont, très engagée durant les trois ans de réalisation, ainsi que d'autres partenaires locaux, publics (Communauté de communes des Monts et Vallées) et privés (mécénat du Crédit Agricole, de la Chambre de Commerce et d'Industrie de Dieppe, de la Chambre française d'Horlogerie et des Micromécaniques).

\section{La sauvegarde dans le musée}

\section{Pérenniser et transmettre les connaissances}

Pour l'équipe du musée, la question essentielle était de réutiliser concrètement cette étude pour améliorer les connaissances sur les collections et d'en rendre les résultats visibles au public.

La première mesure a été la réécriture des cartels du musée grâce aux connaissances et informations nouvellement acquises, notamment en y intégrant des citations directes.

Bien entendu, en parallèle, les fiches de l'inventaire informatisé ont été retravaillées (affinement des datations, des matériaux, des processus de fabrication). Au final, près de trois cents fiches ont ainsi été versées sur la base nationale Joconde, notamment celles concernant des objets horlogers industriels pour lesquels 


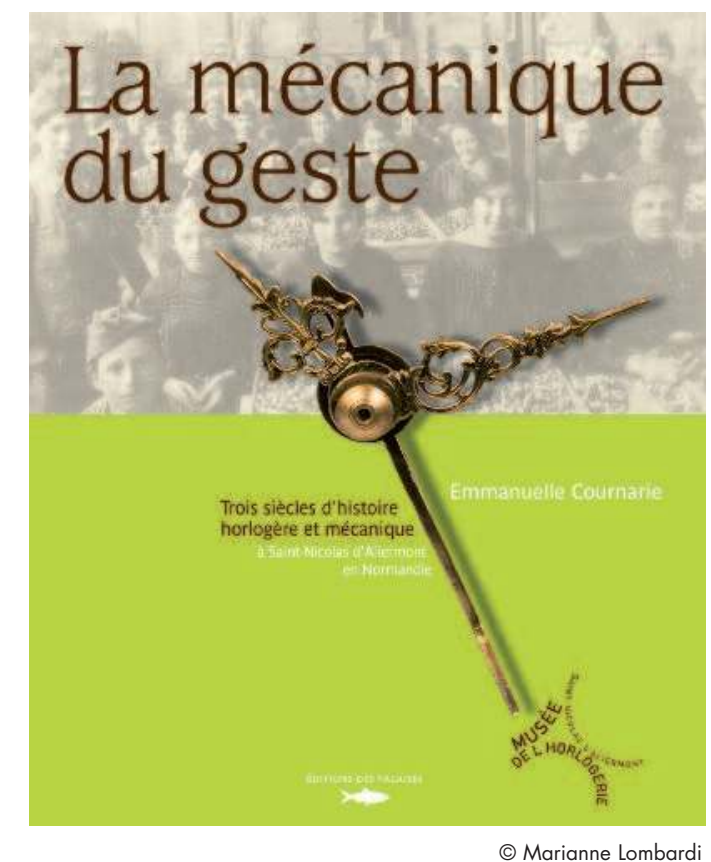

La rédaction d'un ouvrage grand public

Dès le début du projet, nous avions prévu de publier un ouvrage rassemblant les données historiques, techniques et sociologiques recueillies. Son rendu final a été mûri au fil de ce travail sur la mémoire orale. Au début nous pensions à une forme simple, un petit livret accompagné du $\mathrm{CD}$, qui aurait, à travers des témoignages emblématiques, retracé l'histoire de l'Aliermont depuis la Seconde Guerre mondiale. Or, des échanges avec de nombreux autres professionnels ayant mené ce type de travail mémoriel, le manque évident d'un ouvrage synthétique sur l'histoire de la ville, ainsi que celui d'un guide des collections du musée nous ont conduits à élaborer un projet de rédaction d'un ouvrage abondamment illustré plus ambitieux.

À destination des visiteurs mais aussi de la communauté aliermontaise, le livre tel quiil a été réalisé se veut autant un outil de valorisation du patrimoine et des collections du musée qu'un support de la mémoire ouvrière locale garant de sa préservation et de sa pérennisation à travers le temps.

L'ouvrage permet de synthétiser les données historiques existantes et les résultats de la recherche sociologique - en donnant à voir une partie du patrimoine immatériel grâce aux extraits d'entretiens - et met en valeur les collections du musée.

\section{Conclusion}

Pour une structure de petite taille, comme le musée de l'Horlogerie, qui fonctionne avec une équipe réduite et polyvalente, la possibilité d'organiser le projet sur trois ans a permis une mise en œuvre efficace du travail tant au niveau scientifique, qu’administratif et budgétaire. Par ailleurs, la valorisation des résultats de la recherche dans les salles du musée et auprès des habitants s'est traduite par une hausse notable de la fréquentation du musée. Ainsi les personnes interrogées et leurs familles, qui, habituellement, venaient peu ou pas du tout au musée, sont devenues des visiteurs fidèles mais aussi des acteurs importants de l'établissement en effectuant des dons, en se portant volontaires pour assurer des démonstrations techniques et en s'impliquant dans des projets avec le public scolaire (témoignages, visites des anciennes usines avec les classes...).

Dans le prolongement de ce projet mené autour du patrimoine immatériel, d'autres développements sont maintenant envisagés, notamment un partenariat avec les archives départementales de Seine-Maritime pour reverser une partie des entretiens réalisés ainsi qu'une mise en ligne sur le site Internet du musée des données recueillies lors de l'enquête.

\section{Bibliographie}

Berthaux, D. Les récits de vie. Paris : Nathan, 1997.

Kaufmann, J.-C. L'entretien compréhensif. Paris : Nathan, 1996.

Quivy, R. et Van Campenhoudt, L. Manuel de recherche en sciences sociales. Paris : Dunod, 2011.

Verret, M., L'espace ouvrier. Paris : L'Harmattan. 1995.

Verret, M., La culture ouvrière. Paris : L'Harmattan. 1996.

Verret, M., Le travail ouvrier. Paris : L'Harmattan. 1999. 